\title{
3D Matrix Adhesions Mediating Mechanostranduction in
}

\section{hMSC-Collagen Constructs}

\author{
Terry C.W. Li, Dr. Barbara P. Chan \\ Tissue Engineering Laboratory, Department of Mechanical Engineering, \\ The University of Hong Kong, \\ Hong Kong \\ bpchan@hkucc.hku.hk
}

\begin{abstract}
The current study aims to identify the type of cellmatrix adhesions of hMSCs in 3D collagen constructs and to investigate the effects of dynamic compression on the type, morphology and composition of cell-matrix adhesions, particularly to observe whether the compression stimulates the maturation or evolvement of 3D matrix adhesion in hMSCcollagen constructs. Preliminary results demonstrated the colocalization of integrin $\alpha_{5} \beta_{1}$ and fibronectin in cell-matrix adhesions in loaded constructs, partially fulfilling the requirements for 3D matrix adhesion to evolve. In addition, fibronectin was shown to be organized into tiny-dotted adhesions in loaded constructs in a loading duration dependent way, suggesting dynamic compression may be able to mature adhesions in the constructs, hopefully into 3D matrix adhesions. It was also demonstrated that hMSCs plated onto their own cellderived matrices form elongated adhesions which are similar to 3D matrix adhesions formed by fibroblasts. Further characterization on the cell -matrix adhesions of hMSCs in 3D collagen constructs and identification of differences in adhesions between loaded and unloaded constructs are underway.
\end{abstract}

Keywords-component; Mechanotransduction, 3D matrix adhesions, dynamic compression, human mesenchymal stem cells

\section{INTRODUCTION}

Cell-matrix adhesions are bi-directional mechanotransducers between cells and their extracellular matrices (ECM). They are composed of extracellular ligands, transmembrane integrins and intracellular plaque proteins. Three types of cell-matrix adhesions, focal complexes, focal adhesions and fibrillar adhesions have been identified in cells. They are demonstrated to be dynamic, and their sizes, morphologies and molecular compositions are regulated by force [1] (both external force and intracellular force) and substrate pliability [2]. They can affect cellular fate such as migration, proliferation and survival. Recently, a new type of cell-matrix adhesion, namely "3D matrix adhesions" has been identified in fibroblasts plated onto their own cell-derived matrices [2]. These 3D matrix adhesions contribute to distinct cell shape and enhancement of biological activities including migration, attachment and proliferation. Requirements for 3D matrix adhesions include integrin $\alpha_{5}$, fibronectin, threedimensionality, substrate pliability and other matrix components [2].
In this study, we aim to (1) identify the type of cell-matrix adhesions of human mesenchymal stem cells (hMSCs) in 3D collagen constructs and to (2) investigate the effects of dynamic compression on the type, morphology and composition of cell-matrix adhesions, particularly to observe whether the compression stimulates the maturation or evolvement of 3D matrix adhesion in hMSC-collagen constructs and then to (3) examine the roles of $3 \mathrm{D}$ matrix adhesions in regulating stem cell fate.

\section{METHOD}

\section{A. Compression loading system}

Compression loading system was established previously by our group [3]. Briefly, a pair of micromanipulators (MP-285; Sutter Instrument, Novato, CA) was coupled to an inverted research microscope (DMIRB; Leica Microsystems, Wetzlar, Germany) equipped with heat-generating plate (Leica Microsystem). A pair of compression platens was modified from metal spatulas and affixed to the micromanipulators via cylindrical holders. Two-compartment Petri dish with $100 \mathrm{~mm}$ diameter (BD Biosciences, San Jose, CA) was used as the sample holder and was affixed to the heat plate, which was adjusted to $37{ }^{\circ} \mathrm{C}$ during compression loading. Disc shaped samples subjected to the same loading condition were placed between the partition walls of the two-compartment Petri dish. A digital camera (CoolSNAP ES) was used to monitor the real time deformation of the constructs during loading.

\section{B. Culture of hMSCs}

hMSCs were cultured in Dulbecco's modified Eagle's medium-low glucose (Gibco, Grand Island, NY) supplemented with $10 \%$ fetal bovine serum (Gibco), 100U/ml penicillin (Gibco), $100 \mathrm{mg} / \mathrm{ml}$ streptomycin (Gibco), and $2 \mathrm{mM}$ L-glutamine (Gibco), and incubated in a humidified condition with $5 \% \mathrm{CO}_{2}$ at $37^{\circ} \mathrm{C}$. Culture medium was changed every 3 to 4 days. hMSCs at passage 6 were used for collagen encapsulation.

\section{Formation of disc-shaped hMSC-collagen constructs}

Disc-shaped hMSCs-collagen constructs $(1 \mathrm{E}+6$ or $5 \mathrm{E}+5$ cells $/ \mathrm{ml}$, col. conc: $2 \mathrm{mg} / \mathrm{ml}, 100 \mu 1 /$ droplet) were formed in custom-made cylindrical containers using the 
microencapsulation technique developed in our laboratory [4]. A custom-made cylindrical container with non adhesive surface was used to cast the constructs. Rat-tail collagen type I solution was neutralized with sodium hydroxide before mixing with single-cell suspension of hMSC in culture medium. Final cell densities of $1 \mathrm{E}+6$ or $5 \mathrm{E}+5$ cells $/ \mathrm{ml}$ and collagen concentration of $2 \mathrm{mg} / \mathrm{ml}$ were achieved upon dilution of hMSC-collagen mixture by culture medium. Collagen gelation was prevented by keeping the mixture in an ice-bath. Aliquots of $100 \mu \mathrm{l}$ of collagen mixture were pipetted into the cylindrical containers to form the disc-shaped hMSC-collagen constructs. Bottoms of 24-well plate were covered by parafilm to prevent migration of hMSCs from the constructs to the culture wells. The constructs were cultured and allowed to contract for 4 days before compression.

\section{Loading conditions}

Unidirectional, dynamic axial compression was applied to the constructs at frequency of $1 \mathrm{~Hz}$. The constructs were preloaded with $10 \%$ static deformation and followed by cyclic compression of another $10 \%$ peak-to-peak deformation. Two different loading patterns were attempted: (A) 7-day compression: $1 \mathrm{hr}$ per day for 7 consecutive days, samples fixed after overnight incubation post-compression and (B) 1day compression: continuous $3 \mathrm{hrs}$ and $5 \mathrm{hrs}$ in one day, samples fixed after incubation of $18 \mathrm{hrs}$ post-compression.

\section{E. Preparation of cell-derived matrices and cell-seeding}

To investigate whether hMSCs can form 3D matrix adhesions similar to those formed by fibroblasts, cell-derived matrices were produced in a similar way as previously described [2]. In brief, hMSCs at passage 4 were seeded on glass coverslips at 2e4 cells/well onto 24-well plate and cultured for 15 days. Culture medium was changed every 3 to 4 days. During the final 8 days, ascorbic acid $(50 \mathrm{ng} / \mathrm{ml})$ was added to culture medium. At the $15^{\text {th }}$ day of culture, cell-derived matrices were decellularized using extraction buffer (Triton/ $\mathrm{NH}_{4} \mathrm{OH}$ ) at $37^{\circ} \mathrm{C}$. The newly formed cell-derived matrices were washed three to four times by PBS for $5 \mathrm{~min}$ each. hMSCs at passage 6 was seeded onto these cell-derived matrices and fixed after 1-day culture.

\section{F. Histological processing, immunofluorescence and confocal imaging}

hMSC-collagen constructs were fixed by $4 \%$ paraformaldehyde for 30 or $45 \mathrm{~min}$. They are cryoprotected by immersion in $30 \%$ sucrose overnight and then embed in OCT compound. The tissue blocks are then cut by microtome to produce 20 or $30 \mu \mathrm{m}$ thick frozen sections. Sections were airdried overnight and kept at $-80{ }^{\circ} \mathrm{C}$ until immufluorescent staining.

For indirect immunofluroscence, sections were air-dried and rehydrated in PBS. Sections/fixed cells were blocked in 3\% BSA for $30 \mathrm{~min}$ and then incubated in primary antibodies (anti-integrin $\alpha_{5}$ from eBioscience Cat. No: 14-0496, antifibronectin from Santa Cruz Cat. No: Sc-9068) for $1 \mathrm{hr}$ at

Identify applicable sponsor/s here. If no sponsors, delete this text box. (sponsors) room temperature or overnight at $4^{\circ} \mathrm{C}$ and then washed 3 to 4 times in PBS for 5 min each. Sections/fixed cells were then incubated with secondary antibodies (anti-mouse IgG, antirabbit IgG from Invitrogen) for $45 \mathrm{~min}$, washed 3 to 4 times in PBS. Nuclei were labeled by incubating in DAPI for $20 \mathrm{~min}$. Sections/fixed cells were washed 3 times in PBS and mounted in DAKO fluorescent mounting medium and view under confocal microscope at a magnification of $63 x$. Patches of colocalization of integrins and their ligands indicates formation of cell-matrix adhesions.

\section{RESULT}

A. colocalization of integrin $\alpha_{5}$ and fibronectin was observed in loaded constructs but not in unloaded constructs in 7day compression experiments

hMSCs-collagen constructs expressed integrin $\alpha_{5}$ and fibronectin in both loaded and unloaded constructs (fig. 1 and fig.2). It indicates that hMSCs may want to utilize integrin $\alpha_{5^{-}}$ fibronectin bonds to enhance mechanostransduction between cells and their extracellular matrix. However, colocalization of integrin $\alpha_{5}$ and fibronectin was observed in loaded constructs but not in unloaded constructs (fig.3 and fig.4). This demonstrates that integrin $\alpha_{5}$ and fibronectin form ligandreceptor bonds upon dynamic compression.

B. dynamic compression of longer loading duration leads to assembly of more extracellular fibronectin and more mature adhesions in 1-day compression experiments

To see the effect of sustained loading, 3-hr and 5-hr continuous dynamic compression was applied to hMSCcollagen constructs. Fibronectin was diffusely expressed in unloaded constructs but organized into tiny-dotted adhesions in loaded constructs (fig. 5). As integrin $\alpha_{5}$ is the specific receptor for fibronectin, a possible explanation is that dynamic compression enhances formation of integrin $\alpha_{5}$-fibronectin bonds which lead to subsequent clustering of integrin $\alpha_{5}$ and thus assembly of fibronectin. Fibronectin was still weakly expressed in extracellular region in $3-\mathrm{hr}$ compression group while fibronectin was absent in extraceullar region in 5-hr compression group (fig. 6), providing evidence that dynamic compression of longer loading duration leads to assembly of more extracellular fibronectin and more mature adhesions.

\section{C. hMSCs express 3D matrix adhesions when plated onto their own cell-derived matrices}

To demonstrate capabability of hMSCs to form 3D matrix adhesions, they were plated onto their own cell-derived matrices and culture for 1 day. By fibronectin staining, it was shown that hMSCs can form adhesions which possess a characteristic elongated morphology similar to 3D matrix adhesions formed by fibroblasts (fig. 7). This suggests that hMSCs are capable of expressing 3D matrix adhesions. 


\section{DISCUSSION}

Based on the current results, adhesions other than focal adhesions and focal complexes (may be fibrillar adhesions or 3D matrix adhesions or else) are observed in loaded constructs. Different loading patterns will be attempted to observe how dynamic compression can affect the maturation of adhesions. As 3D matrix adhesions are also marked by their long and slender appearance (axial ratio of 33) and other molecular requirement (e.g. paxillin), 3D reconstruction of thicker sections (30 to 50 microns) will be attempted to calculate real axial ratios of the adhesions and observe 3D morphology of the adhesions. And immunofluorescence will be applied to identify the presence of other markers of 3D matrix adhesions in hMSC-collagen constructs.

\section{ACKNOWLEDGMENT}

THIS WORK WAS SUPPORTED BY GRANTS FROM AOSPINE (AOSBRC-07-06); THE INNOVATION AND TECHNOLOGY COMMISSION, THE HONG KONG GOVERNMENT (ITS-133-08) AND THE STRATEGIC RESEARCH THEMES OF THE UNIVERSITY OF HONG KONG ON BIOMEDICAL ENGINEERING AND BIONANOTECHNOLOGY.

\section{REFERENCES}

[1] Harjanto, D. and M.H. Zaman, Matrix mechanics and receptor-ligand interactions in cell adhesion. Org Biomol Chem,2010. 8(2): p. 299-304.

[2] Cukierman, E., et al., Taking cell-matrix adhesions to the third dimension. Science,2001. 294(5547): p. 1708-12.

[3] Au-Yeung, K.L., et al., Development of a micromanipulator-based loading device for mechanoregulation study of human mesenchymal stem cells in three-dimensional collagen constructs. Tissue Eng Part C Methods, 2010. 16(1): p. 93-107.

[4] Chan, B.P., et al., Self-assembled collagen-human mesenchymal stem cell microspheres for regenerative medicine. Biomaterials, 2007. 28(31): p. 4652-66.

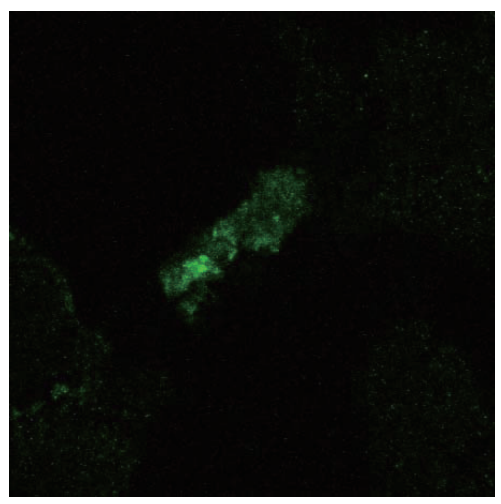

Fibronectin (green)

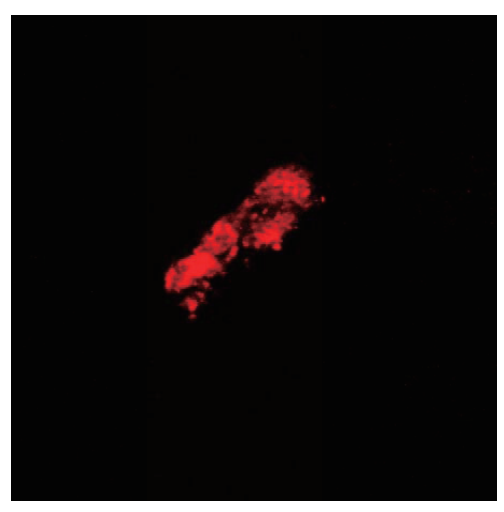

Integrin $\alpha_{5}$ (red)

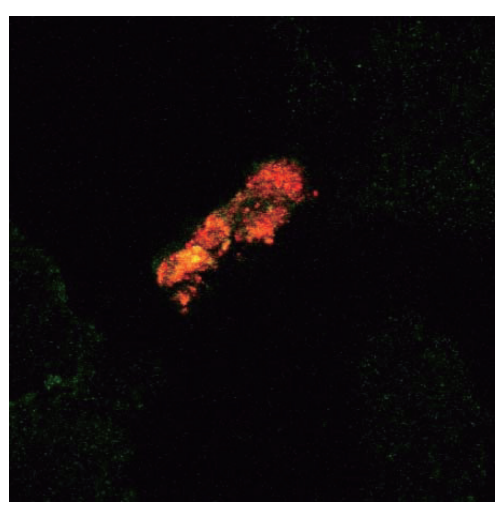

merged

Fig. 3 - Colocalization of integrin $\alpha_{5}$ and fibronectin (red+green $\rightarrow$ yellow) is observed in 7-day compression group

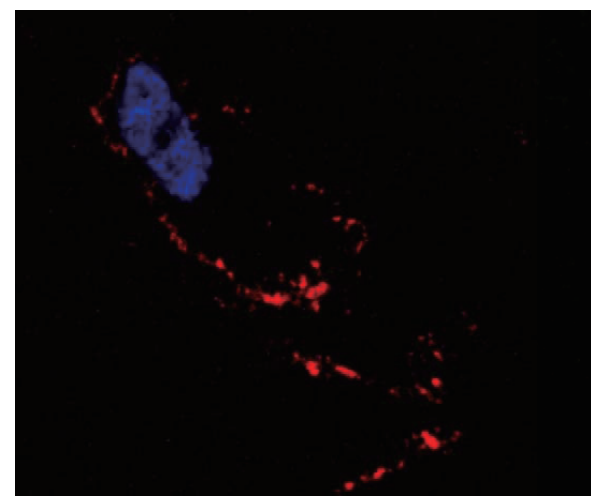

Fig. 4 - integrin $\alpha_{5}$ (red) and nucleus (blue). No specific staining (only weak, diffuse staining, not shown in this photo) was observed for fibronectin (which should be green) in the control group (no compression) 


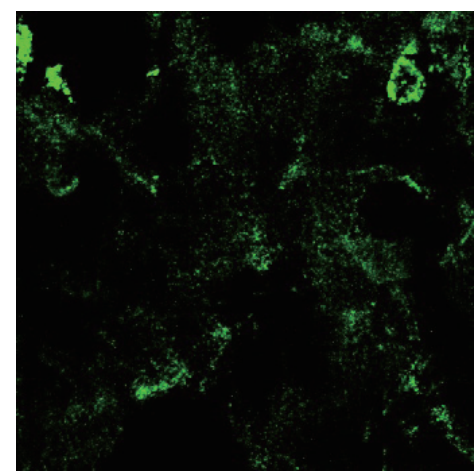

control

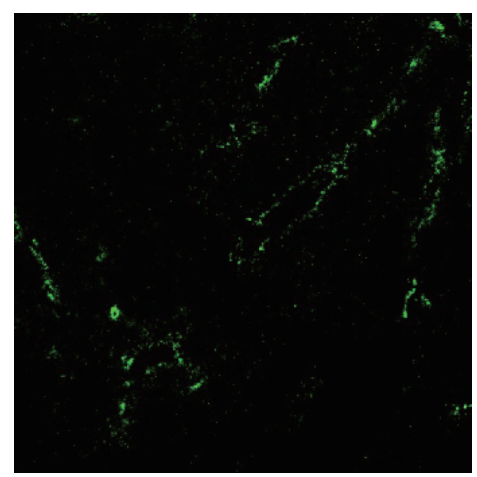

3-hr compression

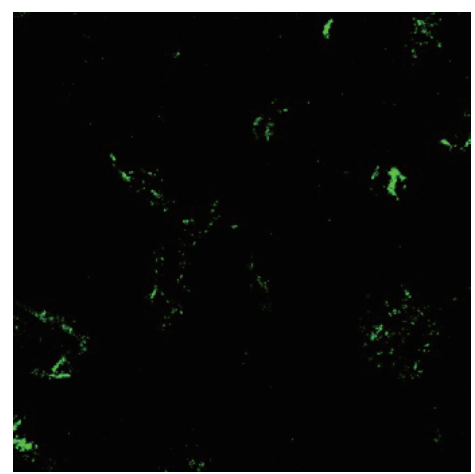

5-hr compression

Fig. 5 - fibronectin was organized into tiny-dotted adhesions in 1-day compression group ( $3 \mathrm{hrs}$ or $5 \mathrm{hrs)} \mathrm{but} \mathrm{diffusely} \mathrm{expressed} \mathrm{in} \mathrm{controls}$

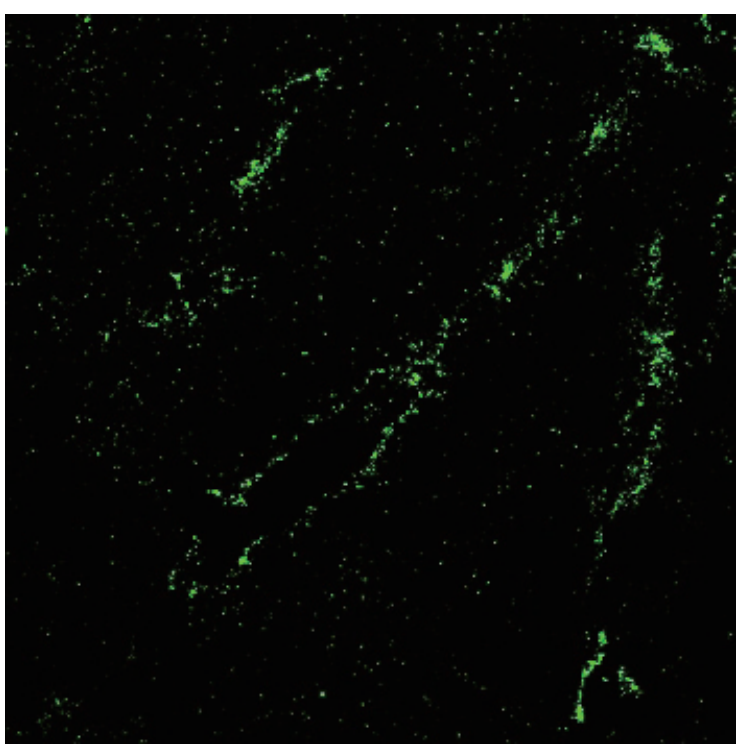

3-hr compression

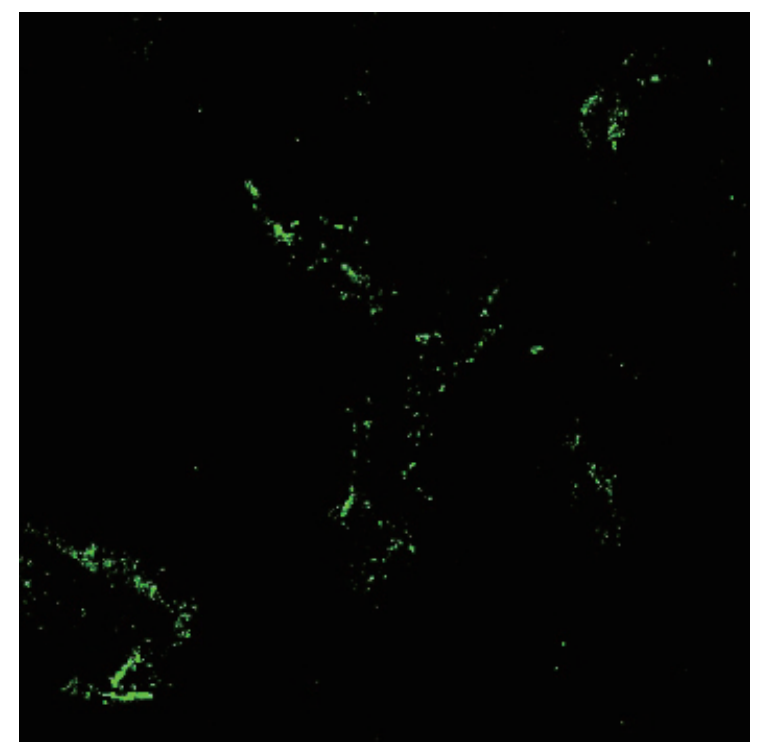

5-hr compression

Fig. 6 - fibronectin was still weakly expressed in extracellular region in 3-hr compression group while fibronectin was absent in extraceullar region in 5-hr compression group.

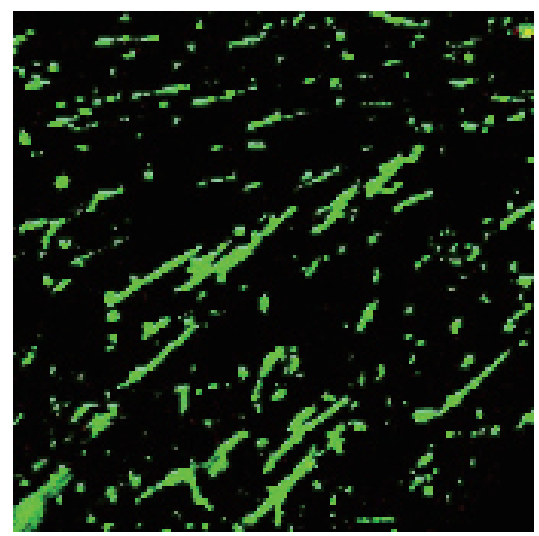

Fig. 7 - adhesions formed by hMSCs when plated onto their own cell-derived matrices for 1 day 